\title{
AnÁlise da PERCEPÇão de COMPETÊnCIA física de CRIANÇAS COM DEFICIÊNCIA MENTAL
}

\author{
EXAMINATION OF PERCEIVED PHYSICAL COM PETENCE OF CHILDREN WITH MENTAL
}

RETARDATION

\author{
Larissa Daniele Rubira STRIOTO ${ }^{1}$ \\ Lenamar Fiorese VIEIRA ${ }^{2}$ \\ José Luiz Lopes VIEIRA ${ }^{3}$
}

RESUM 0: o objetivo deste estudo descritivo foi analisar a percepção de competência física de crianças com deficiência mental (DM) moderada em relação a diferentes idades e gêneros, bem como comparar a percepção de competência física dos praticantes e não-praticantes de atividades físicas ou esportivas. Foram sujeitos 76 crianças. Como instrumento foi utilizada a Pictorial Scale of Perceived Physical Competence for Children with $M$ ental Retardation, um questionário com questões referentes à prática de atividades físicas e/ ou desportivas fora do ambiente escolar e outro com questões referentes às atividades realizadas dentro do ambiente escolar. Os resultados sugerem que: crianças com deficiência mental moderada apresentam alta percepção de competência; não houve diferença significativa entre gêneros; crianças com idade de 12 anos apresentaram níveis de percepção de competência física menor do que a das crianças de 7, 8 e 9 anos $(p=0,01)$; crianças que praticam exercícios físicos ou esportes têm percepção de competência física menor do que os não-praticantes. Assim conclui-se: a prática de exercícios físicos/ esportivos evidencia-se como elemento interveniente na elaboração dos critérios de julgamento da percepção de competência das crianças, auxiliando na construção de parâmetros avaliativos mais precisos e realistas.

PALAVRAS-CHAVE: educação especial; percepção de competência; crianças; deficiência mental.

ABSTRACT: the objective of this descriptive study was to analyze the perceived physical competence of children with mild mental retardation in relation to different ages and genders as well as to compare the perceived physical competence of children that practice and children that don't practice physical or sports activities. The sample consisted of 76 children. The instruments used were the Pictorial Scale of Perceived Physical Competence for Children with $\mathrm{M}$ ental Retardation, a questionnaire assessing the practice of physical activities or sports out of school and another questionnaire assessing the activities in school. The results suggested that children with mild mental retardation have high perceived physical competence; no significant difference emerged among the genders; 12-year-old children presented lower perceived physical competence than children who were 7, 8 and 9 years old; children that engage in physical exercises or sports have smaller perceived physical competence than those that don't engage in physical activities. The findings suggested that the practice of physical or sports exercises can be seen as an aspect responsible for children's judgment criteria for perception of competence, helping to build more exact and realistic evaluation parameters.

KEYWORDS: Special Education; Perceived Competence; Children; Mental Retardation.

\footnotetext{
${ }^{1}$ Mestranda em Ciência do Movimento Humano, Universidade Estadual de Maringá (UEM), Departamento de Educação Física - larissastrioto@hotmail.com

2 Doutora em Educação Física. Professora do Programa de Pós-Graduação Associado UEM/ UEL, Departamento de Educação Física - Ifvieira@uem.br

${ }^{3}$ Doutor em Educação Física. Professor do Programa de Pós-Graduação Associado UEM/ UEL, Departamento de Educação Física - jllvieira@uem.br
} 


\section{INTRODUÇÃo}

A percepção de competência pode ser definida como sendo os julgamentos dos indivíduos em relação as suas habilidades em domínios específicos (físico, acadêmico, social, dentre outros) e vem sendo assunto prevalente em várias pesquisas (VIEIRA, 1993; VIEIRA et al., 1997; WEISS et al., 1997; EBBECK; WEISS, 1998; GÓMEZ et al., 2006; SOLLERHED et al., 2007; CARROLL; LOUMIDIS, 2008;). Muitas destas tem como base o modelo de percepção de competência de Harter (1978), que propõe que a percepção que o indivíduo tem desi mesmo influencia diretamenteo nível demotivação, autoestima e desenvolvimento social.

Pesquisas sugerem queos padrões de motivação adotados por crianças na participação esportiva, na atividade física ou mesmo em outros contextos de aprendizagem, parecem ser fortemente influenciados pelas percepções de competência que estas evidenciam (HARTER, 1978; RUDISILL, 1989; VALENTINI, 2002;). Por exemplo: uma criança que se auto-percebe como tendo capacidade para jogar futebol, tende a continuar a participar nesse tipo de atividade, mesmo em face de dificuldades. Já uma criança que se sente incompetente, tem maior probabilidade de abandonar a atividade.

Nesse sentido, estudos têm investigado a relação entre a prática de atividade física e a percepção de competência em indivíduos sem deficiência, porém os resultados são inconsistentes. PAPAIOAN NOU (1997) verificou esta relação, por outro lado, Ulrich (1987) não constatou a rel ação entre essas variáveis. Ulrich (1987) examinou a inter-relação entre as variáveis percepção de competência física, competência motora atual e a participação em esportes organizados em crianças da pré-escola a quarta séries, encontrando que a percepção de competência não é significativamente relacionada com suas participações em programas de esporte organizado. Já os resultados do estudo de Papaioannou (1997), mostraram que os indivíduos que estavam engajados em atividades esportivas e/ ou recreacionais fora do ambiente escolar apresentaram escores mais altos nas medidas de percepção de competência física do que indivíduos que não realizavam estas atividades.

Roberts et al. (1981) também avaliaram a percepção de competência e o envolvimento em atividades esportivas, concluindo que, participantes de esportes organizados apresentavam percepção de competência mais elevada. Estes resultados são destacados na maioria de outros estudos, além de reportarem o aumento da autoestima (FELTZ; PETLICHKOFF, 1983; DALEY; BUCHANAN, 1999; CARROLL; LUMIDIS, 2008).

Pesquisas prévias têm mostrado ainda o impacto das diferenças entre gênero na percepção de competência, sendo que os meninos revelam níveis mais al tos quando comparados às meninas (HARTER, 1982; ULRICH, 1987; RUDISILL, 1993; BORTOLI; ROBAZZA, 1997; RAUDSEPP; LIBLIK, 2002). 
Entretanto, apesar da vasta literatura na área da percepção de competência esta mesma ênfase não tem sido dada no campo da deficiência mental (BURACK et al., 1998). N esta área al gumas temáticas têm sido estudadas, como afetividade (GUHUR 2007), habilidades sociais e problemas de comportamento (ROSIN-PIN OLA, 2007), qualidade de vida (SAVIANI-ZEOTI, 2008), dentre outras.

Sendo assim, surgiu o questionamento a respeito do comportamento de crianças com DM: Qual a percepção de competência física de crianças com deficiência mental de 7 a 12 anos de idade?

Neste contexto, o objetivo deste estudo foi analisar a percepção de competência física de crianças com deficiência mental em relação a diferentes idades e gêneros, bem como comparar a percepção de competência dos praticantes e não-praticantes de exercícios físicos ou esportes.

2 Metodologia

\subsection{SuJeitos}

Fizeram parte do estudo 76 crianças com deficiência mental (53 do sexo masculino e 23 do sexo feminino), com idade entre 7 e 12 anos $(M=10,26$, $\mathrm{DP}=1,75)$. Todas de escolas de Educação Especial de três cidades do Noroeste do Paraná. Como critérios de seleção foram utilizados: ter idade entre 7 e 12 anos, não terem deficiência física e a classificação de deficiência mental moderada, diagnosticada pelas próprias escolas.

\subsection{InSTRUMENTOS}

\subsubsection{PercepÇão de Com petência física}

Foi avaliada utilizando-se a PSPPCCMR - Pictorial Scale of Perceived Physical Competence for Children with M ental Retardation (ULRICH; COLLIER, 1990), que é uma versão modificada da Pictorial Scale of Perceived Competence for Y oung Children de Harter ePike's (1984). A PSPPCCMR foi validada para crianças com idade entre 7 e 12 anos de idade com deficiência mental moderada. Consiste de 10 itens (saltar à distância, correr, rebater uma bola lançada, arremessar uma bola de basquete, chutar, arremessar uma bola no alvo, equilibrar, agarrar, pular corda e quicar uma bola). São apresentadas em pranchas contendo duas figuras colocadas uma ao lado da outra; uma figura corresponde a uma criança competente e outra a uma criança não tão competente. A criança primeiramente deve selecionar qual figura mais se parece com ela e então decidir se é muito ou pouco parecida com a criança da figura. Os itens são pontuados seguindo uma escala de 4 pontos, onde 1 corresponde a uma baixa competência e 4 a uma alta competência. Não é necessário que se saiba ler para responder a esta avaliação. A figura 1 é um exemplo dos itens da escala. São utilizadas pranchas com figuras retratando meninos e meninas, cada uma para avaliar o gênero correspondente. 

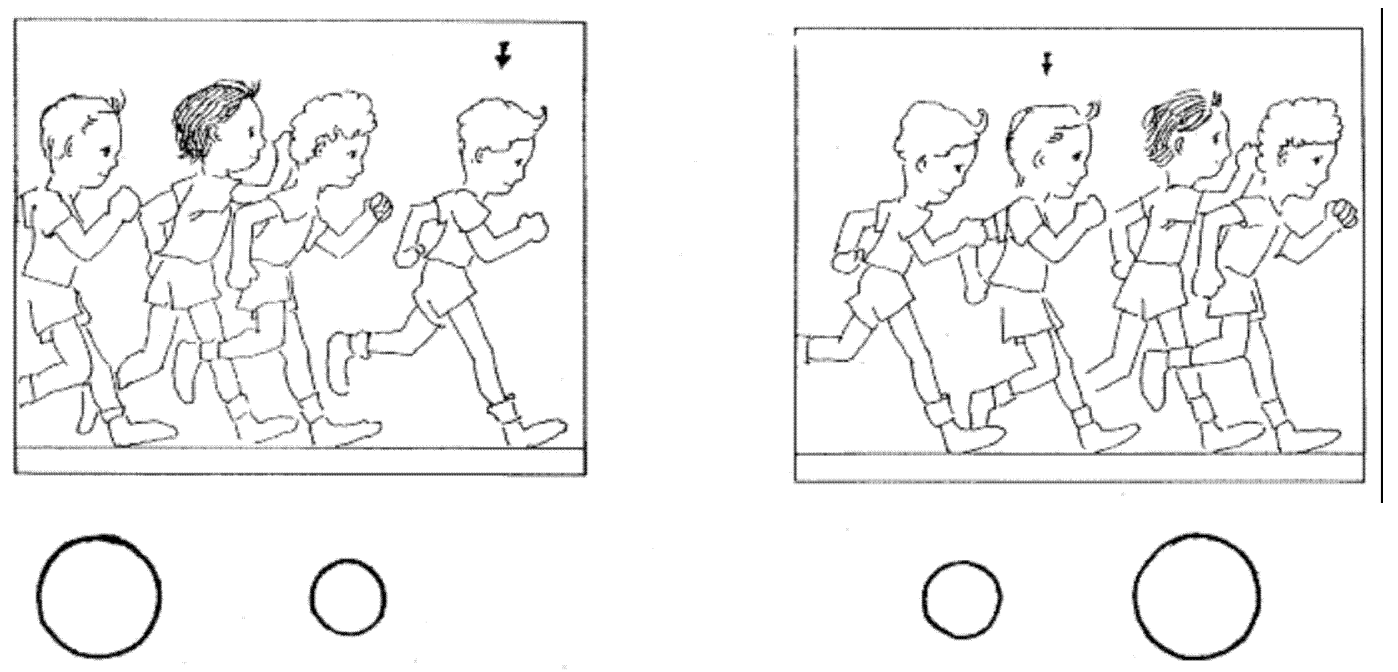

Figura 1 - Exemplo de item das pranchas masculinas da Pictorial Scale of Perceived Physical Competence de Ulrich e Collier (1990).

\subsubsection{ParticipaÇão em exercícios físicos/esportivos fora do ambiente escolar}

Aplicou-se um questionário para investigar a participação das crianças em exercícios físicos fora da escola. Este foi uma adaptação do questionário de N eto (1997). Uma das alterações foi a inclusão de questões referentes à prática de exercícios físicos, já que no original referia-se apenas a esportes. Além disso, no protocolo original, as crianças deveriam responder às questões, porém nesteestudo, os pais ou responsáveis responderam pelas crianças.

O questionário foi composto de 12 questões abertas e fechadas com questões relativas à prática ou não de al gum esporte ou exercício físico, freqüência semanal e justificativa de não participar de exercícios físicos ou esportes.

\subsubsection{Participação em atividades no ambiente escolar}

Um questionário foi elaborado aos coordenadores ou responsáveis pelas atividades extraclasse das escolas contendo questões referentes à prática de atividades variadas, como Fisioterapia, Educação Física, Esportes, ou outros exercícios físicos como por exemplo hidroginástica ou musculação. As questões eram apresentadas em forma de check list. Caso a resposta fosse afirmativa, respondiam qual eram os esportes e/ ou exercícios físicos praticados e qual a freqüência semanal que eram realizados. No entanto, nenhuma criança real izava exercícios físicos ou esportivos dentro do ambiente escolar. 


\subsection{Procedimentos}

Inicialmente, as escolas foram contactadas e assinaram o termo de consentimento, autorizando o estudo.

Posteriormente, as escolas enviaram aos pais ou responsáveis o convite para participação da pesquisa contendo a data, o local e o horário para esclarecimentos do estudo e preenchimento dos questionários. Aos pais que não compareceram foi enviado o questionário pela caderneta das crianças ou entregue por um funcionário da escola pessoalmente.

Finalizado este processo, a Escala de Percepção de Competência Física foi administrada nas crianças na própria escola em sala de aula cedida pela coordenação. Cada criança foi avaliada individualmente e o tempo da aplicação foi em média de 8 min.

Foram considerados praticantes de exercícios físicos/ esportivos, aqueles que realizassem ao menos uma vez por semana algum exercício físico ou esporte dentro ou fora do ambiente escolar.

O projeto teve aprovação do Comitê de Ética sob parecer nº 175/ 2007.

\subsection{Análise estatística}

Para testar a normalidade dos dados e assim definir quais seriam os testes estatísticos foi utilizado o teste de Shapiro-Wilk. Devido à distribuição nãonormal dos dados, o teste Mann-Whitney foi utilizado para a comparação entre os grupos praticantes e não-praticantes de exercício físico/ esporte e entre os gêneros. Já para verificar possíveis diferenças entre as idades foi utilizado o teste de Kruskal-Wallis com post-hoc de Games Howell. Foi adotado P <0,05.

\section{Resultados e discussões}

A primeira análise foi projetada a determinar a percepção de competência física de crianças com deficiência mental. Como apresentado na Figura 2, as crianças deste estudo revelaram alta percepção de competência física, pelo fato de a mediana $(3,6)$ estar acima de 2,5 que é o ponto médio na escala de percepção de competência. Estes resultados suportam os achados em pesquisas prévias com deficientes mentais (ULRICH; COLLIER, 1990; YUN; ULRICH, 1997; SOKOLOWSKI et al., 2000). Baseando-se na teoria de Harter (1978), a qual destaca a percepção de competência como sendo estruturada em quatro fatores: as experiências passadas, dificuldades ou desafios associados com o resultado da tarefa; suporte e interação pessoal com outros que são significativos para a criança e a motivação intrínseca. Os resultados indicam que os altos escores apresentados podem estar relacionados a estes fatores, já que $92 \%$ das crianças realizavam aulas de Educação Física dentro do ambiente escolar apenas uma vez por semana com duração aproximada de 50 minutos. A penas $17 \%$ realizavam 
alguma atividade esportiva fora do contexto escolar, com freqüência de pelo menos uma vez por semana. As justificativas dadas pelos pais ou responsáveis para a não realização de atividades foi a falta de recursos financeiros (50\%), por não haver atividades para crianças com deficiência (52\%), não podem levar as crianças até o local (36\%), o local onde é oferecido a atividade fica muito distante da moradia (41\%), dentre outras (32\%).

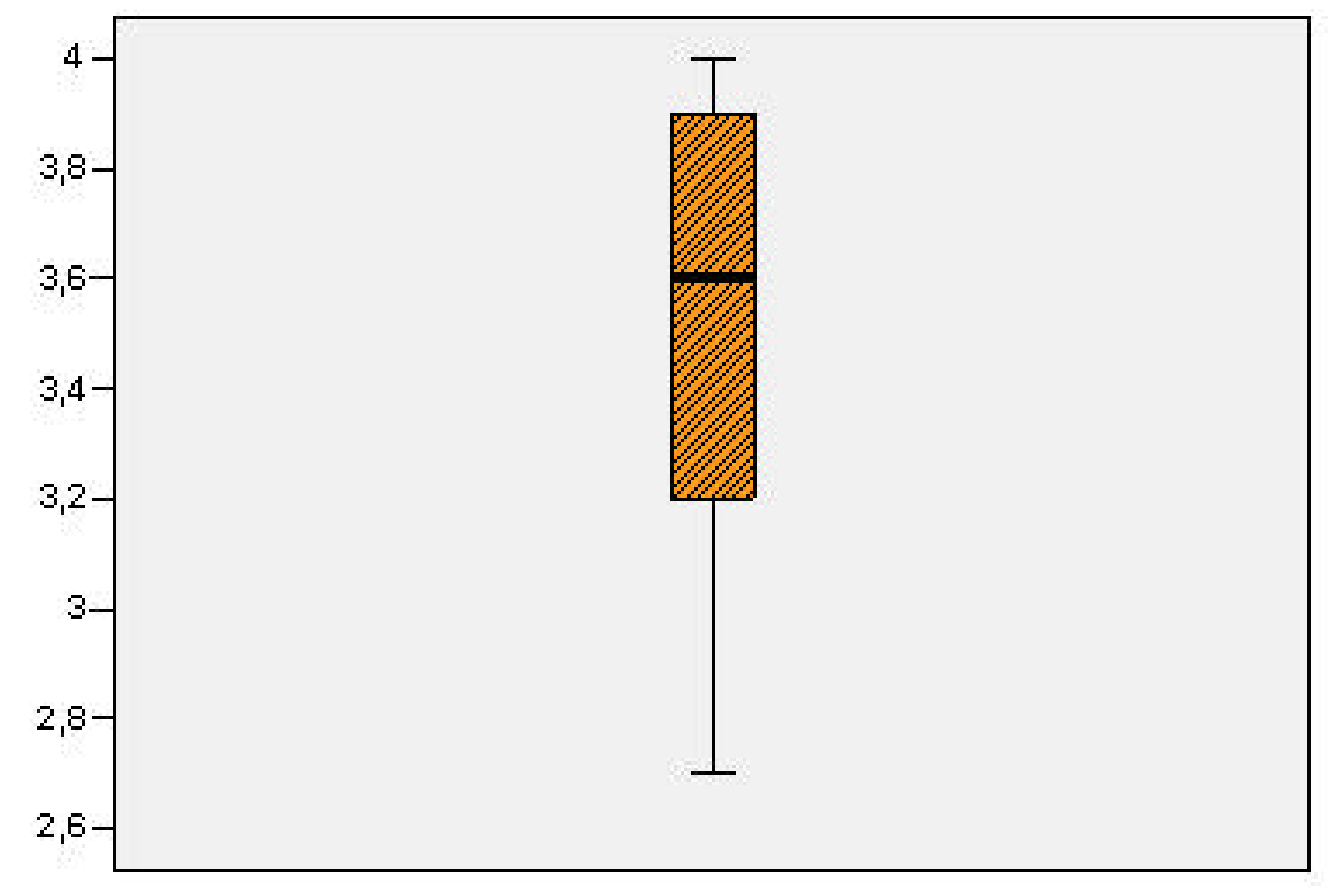

Figura 2 - Percepção de competência física de crianças com deficiência mental

Como é possível observar na Tabela 1, os valores da percepção de competência física permaneceram altos nos diferentes grupos etários, embora possa se perceber que os valores tendem a diminuir quando comparadas as crianças de 7-9 anos e as crianças de 10-12 anos. A aplicação do teste de KruskalWallis revelou que havia diferença significativa na percepção de competência física entre os grupos $(P<0,0001)$.

Para localizar qual idade se difere das outras na percepção de competência física, foi conduzida uma análise post-hoc. Entre as crianças de 7, 8 e 9 anos não foram encontradas diferenças significativas. No entanto, quando comparadas às idades superiores (10 e 12 anos), encontrou-se diferença significativa. Com 12 anos a percepção decompetência física foi significativamente menor do que das crianças com 7, 8 e 9 anos. 
A pesar das crianças terem uma capacidade cognitiva moderada, percebe-se que assim como as crianças sem deficiência, conforme a idade avança, vão criando critérios de julgamento, isto deve-se muito provavelmente ao suporte e interação pessoal com os adultos (HARTER, 1978), em especial com os pais e familiares que oferecem este feedback.

Burack et al. (1998) destacam que até em torno dos 8 anos de idade, as crianças sem deficiência mental ainda não têm seu autoconceito definido. Levando-se em consideração que as crianças com deficiência mental possuem um atraso de dois a quatro anos no funcionamento cognitivo, apenas as mais velhas deste estudo estariam mais aprimoradas para sua autoavaliação.

Crianças mais velhas começam a perceber que não são boas em tudo e também fazem comparações sociais durante a realização das tarefas, enquanto crianças mais novas pensam que são boas em tudo sem considerar quão bem as outras crianças estão realizando (YUN; ULRICH, 1997).

Tabela 1 - Comparação da percepção de competência física em relação à idade das crianças com deficiência mental.

\begin{tabular}{|c|c|c|c|c|}
\hline (I) Idade & $n$ & Mediana (25-75) & (J) Idade & Sig. \\
\hline \multirow[t]{5}{*}{7 anos } & 8 & $4(3,8-4)$ & 8 anos &, 424 \\
\hline & & & 9 anos & 1,000 \\
\hline & & & 10 anos &, $001 *$ \\
\hline & & & 11 anos &, 421 \\
\hline & & & 12 anos &, $000^{*}$ \\
\hline \multirow[t]{5}{*}{8 anos } & 7 & $3,8(3,6-3,9)$ & 7 anos & ,424 \\
\hline & & & 9 anos &, 327 \\
\hline & & & 10 anos & ,019 \\
\hline & & & 11 anos &, 895 \\
\hline & & & 12 anos &, $001 *$ \\
\hline \multirow[t]{5}{*}{9 anos } & 9 & $4(3,9-4)$ & 7 anos & 1,000 \\
\hline & & & 8 anos &, 327 \\
\hline & & & 10 anos &, $001 *$ \\
\hline & & & 11 anos &, 389 \\
\hline & & & 12 anos &, $000^{*}$ \\
\hline \multirow[t]{5}{*}{10 anos } & 15 & $3,2(3,1-3,6)$ & 7 anos & ,001* \\
\hline & & & 8 anos & ,019 \\
\hline & & & 9 anos &, $001 *$ \\
\hline & & & 11 anos &, 714 \\
\hline & & & 12 anos & 1,000 \\
\hline \multirow[t]{5}{*}{11 anos } & 7 & $3,6(3,4-3,9)$ & 7 anos & ,421 \\
\hline & & & 8 anos &, 895 \\
\hline & & & 9 anos &, 389 \\
\hline & & & 10 anos &, 714 \\
\hline & & & 12 anos &, 534 \\
\hline \multirow[t]{5}{*}{12 anos } & 14 & $3,2(2,9-3,6)$ & 7 anos & ,000* \\
\hline & & & 8 anos &, $001 *$ \\
\hline & & & 9 anos &, $000^{*}$ \\
\hline & & & 10 anos & 1,000 \\
\hline & & & 11 anos &, 534 \\
\hline
\end{tabular}

$* \mathrm{p}<0,05$ 
Outro aspecto examinado no estudo foi a diferença da percepção de competência física entre as crianças com deficiência mental praticantes (17\%) e as não-praticantes (83\%) de exercícios físicos ou esportes. A hipótese, baseada nas pesquisas prévias, era queindivíduos que praticassem atividades teriam maior percepção de competência física. No entanto, o teste de Mann-Whitney revelou que crianças que praticavam exercícios físicos ou esportes, fora do ambiente escolar, apresentaram níveis mais baixos de percepção de competência ( $P=0,008$ ).

Uma explicação para estes resultados é que as crianças que praticam exercícios físicos ou esportes passam a introjetar critérios de julgamento mais precisos e melhoram a percepção de sua competência física, sendo esta mais condizente com a realidade. Frente às dificuldades, percebem que muitas vezes não são "tão boas" quanto jul gavam ser antes da oportunidade de praticar al gum exercício físico ou esporte. Esta colocação vem ao encontro do que Harter (1978) e Rudisill (1989) destacam, que quando são dadas oportunidades de vivenciar práticas motoras, as crianças passam a ter melhor precisão na construção de parâmetros avaliativos.

Estes resultados destacam que as experiências com exercícios físicos e esportivos auxiliam as crianças na elaboração de critérios de julgamento, auxiliando na sua percepção de competência. Para Harter (1978) e Rudisill (1989), a falta de oportunidades de vivenciar exercícios físicos variados é um fator interveniente na construção de parâmetros avaliativos mais precisos.

O estudo de Sokolowski et al. (2000) investigou os efeitos da participação em esportes na percepção de competência de adolescentes com deficiência mental e demonstrou resultados semelhantes, a percepção de competência diminuía conforme a participação nos esportes aumentava.

N os estudos realizados em crianças sem deficiência os resultados são divergentes. Indivíduos que não eram envolvidos em nenhum tipo de atividade esportiva fora da escola apresentaram escores mais baixos na competência física e acadêmica, quando comparados com os que estavam envolvidos em atividades esportivas ou atividades físicas recreacionais (PAPAIOAN NOU, 1997).

Efetuadas as comparações entre os gêneros, pode-se notar que não houve diferença significativa na percepção de competência física ( $P=0,069)$. Isto vem ao encontro de estudos real izados com crianças sem deficiência, os quais não observaram diferenças nas percepções entre os gêneros para esta faixa etária (ULRICH, 1987; GOODWAY, RUDISILL, 1996, 1997; VALENTINI, 2002). Porém, outras pesquisas mostram resultados diferentes, indicando distinção entre os gêneros (FELTZ; BROWN, 1984; ULRICH ,1987; RUDISILL et al.,1993; BORTOLI; ROBAZZA,1997; LINDWALL; HASSMÉN, 2004; MOREN O; CERVELLÓ, 2005;). 
Neste sentido, novas investigações serão necessárias buscando observar se os níveis altos de percepção de competência estariam relacionados ao desempenho atual dos indivíduos ou a superestimação de suas competências. Além disso, realizar novas pesquisas com faixas etárias superiores a fim de conhecer melhor o desenvolvimento da percepção de competência física de indivíduos com deficiência mental moderada ao longo dos anos.

Uma limitação deste estudo foi o fato de não ter sido investigado o nível socioeconômico dos indivíduos, para quefossepossível comparar eidentificar possíveis diferenças na percepção de competência, considerando as oportunidades para realização de exercícios físicos ou esportivos.

\section{CONCLUSÃo}

Foi possível considerar, mediante a observação destes resultados, que as crianças com deficiência mental moderada evidenciaram alta percepção de competência física.

Quando oportunidades foram propiciadas fora do ambiente escolar, permitindo ao indivíduo experimentar, conhecer, tentar realizar novas tarefas, que foi o caso da prática de exercícios fiscos ou atividades esportivas, esta percepção apresentou-se significativamente menor, sendo este fator importante na elaboração de critérios de julgamento. Considerando a importância da percepção de competência na motivação e autoconceito da criança para conquistas e participações em exercícios físicos e/ ou esportes, torna-se relevante oportunizar às crianças experiências motoras, visando auxiliá-las nos seus critérios para avaliar sucesso einsucesso, tornando-as mais conscientes de sua competência física, já que a realização de exercícios físicos e/ ou esportivos Ihes permite o conhecimento dos desafios e dificuldades das tarefas propostas, auxiliando na construção de parâmetros avaliativos mais precisos e realistas de suas competências. 


\section{Referências}

BORTOLI, L.; ROBAZZA, C. Italian version of theperceived physical ability scale. Perceptual and M otor Skills. v. 85, n.1, p.187-192, 1997.

BURACK, J. A.; HODAPP, R. M.; ZIGLER, E. H andbook of mental retardation and development. Cambridge: CambridgeUniversity Press, 1998.

CARROLL, B.; LOUMIDIS, J. Children's perceived competenceand enjoyment in physical education and physical activity outsideschool. European physical education review, v. 7, n. 1, p. 24-43, 2008.

DALEY, A. J.; BUCHANAN, J. A erobic dance and physical self-perceptions in female adolescents: some implications for physical education. Research $Q$ uarterly for Exercise and Sport, v. 70, n. 2, p. 196-200, 1999.

EBBECK, V.; WEISS, M. Determinants of children's self-esteem: An examination of perceived competence and affect in sport. Pediatric ExerciseScience, v. 10, p. 285-298, 1998.

FELTZ, D. L.; BROWN, E.W. Perceived competence in soccer skills among young soccer players. Journal of Sport and Exercise Psychology,v. 6, p. 385-394, 1984.

FELTZ, D. L.; PETLICHKOFF, L. Perceived competence among interscholastic sport partici pants and dropouts. Canadian J ournal A pplied Sport Science, v. 8, n. 4, p. 231-235, 1983.

GÓMEZ, P. H.; MURCIA, J. A. M.; GARCÍA, P. L. R. Relación de la competencia motriz percibida con la prática físico-desportiva. Revista de Psicología del D eporte, v. 15, n. 2, p. 219 231, 2006.

GOODWAY,I.D.; RUDISILL, M. E. Influenceof a motor skill intervention programon perceived competence of at-risk A frican A merican preschoolers. A dapted Physical A ctivity Q uarterly, v. 19, p. 288-301, 1996.

GOODWAY, J. D.; RUDISILL, M. E. Perceived physical competenceand actual motor skill competence of A frican A merican preschoolchildren. A dapted Physical A ctivity Q uarterly, $\mathrm{v}$. 14, p. 314-326, 1997.

GUHUR, M. L. P. A manifestação da afetividadeem sujeitos jovens eadultos com deficiência mental: perspectivas de Wallon e Bakhtin. Revista Brasileira de Educação Especial, v.13, n.3, p.381-398, 2007.

HARTER, S. Effectance motivation reconsidered. H uman D evelopment, v. 21, p. 34-64, 1978.

.The perceived competencescalefor children. Child D evel opment, v. 53, p. 87-89, 1982.

HARTER, S.; PIKE, R. The pictorial scal e of perceived competenceand social acceptancefor young children. Child D evelopment. v. 55, p.1969-1982, 1984.

LINDWALL, M.; HASSMÉN, P. Therole of exerciseand gender for physical self-perception and importance ratings in Swedish university students. Scandinavian Journal of M edicine \& Science in Sports, v. 14, n. 6, p. 373-380, 2004.

MORENO, J. A.; CERVELLÓ, E. Physical self-perception in Spanish adolescents: Effects of gender and involvement in physical activity. Journal of H uman M ovement Studies, v. 48, p. 291311, 2005. 
NETO, C. A. F. Jogo e desenvolvimento da criança. Lisboa: Universidade Técnica de Lisboa: Edições FMH, 1997.

PAPAIOANNOU, A. Perceptions of motivational climate, perceived competence, and motivation of students of varying ageand sport experience. Perceptual and M otor Skills, v. 88, n. 2, p. 419-430, 1997.

RAUDSEPP, L.; LIBLIK, R. Relationship of perceived and actual motor competencein children. Perceptual and motor skills, v. 94, n. 2, p. 1059-1070, 2002.

ROBERTS, G.C.; KLEIBER, D. A.; DUDA, J.L. An analysis of motivation in children's sport: Therole of perceived competencein participation. J ournal of Sport P sychology, v. 3, p. 206-216, 1981.

ROSIN-PINOLA, A. R.; DEL PRETTE, Z. A. P.; DEL PRETTE, A. Habilidades sociais e problemas de comportamento de alunos com deficiência mental, al to e baixo desempenho acadêmico. Revista Brasileira de Educação Especial, v.13, n.2, p.239-256, 2007.

RUDISILL, M. E. Influence of perceived competence and causal dimension orientations on expectations, persistence and performance during percei ved failure. Research Q uarterly for Exerciseand Sport, v. 60, p. 166-175, 1989.

RUDISILL, M.E.; MAHAR, M.T; MEANEY, K. S. Therelationship between children perceived and actual motor competence. Perceptual and M otor Skills, v.76, p. 895-906, 1993.

SA VIANI-ZEOTI, F.; PETEAN, E. B. L. A qualidadedevida depessoas com deficiência mental leve. Psicologia: Teoria e Pesquisa, v.24, n.3, p.305-311, 2008.

SOKOLOWSKI, M. et al. Effects of integrated sport participation on perceived competence for adolescents with mental retardation. A dapted Physical A ctivity Q uarterly, v.17, p. 208-221, 2000.

SOLLERHED, A. C. et al. Factors associated with young children's self-perceived physical competenceand self-reported physical activity. H ealth Education Research, v.8, p. 20-32, 2007.

ULRICH, B. Perceptions of physical competence, motor competence, and participations in organized sport: Their interrelationships in young children. Research Q uartely for Exercise and Sport, v.58, p. 57-67, 1987.

ULRICH, D. A.; COLLIER, D. H. Perceived physical competence in children with mental retardation: modification of a pictorial scale. A dapted P hysical A ctivity Q uarterly, v. 7, p. 336354, 1990.

VALENTINI, N. C. Percepções de competência e desenvolvimento motor de meninos e meninas: um estudo transversal. M ovimento, Porto A legre, v. 8, n. 2, p. 51-62, 2002.

VIEIRA , L. F. Relação entrea percepção decompetência deatletas adolescentes eseus motivos para a prática esportiva. R evista da Educação Física da U em, v. 4, n. 1, p. 40-44, 1993.

VIEIRA, L. F.; VIEIRA, J. L. L.; KREBS, R. J. A nálise da percepção decompetência no contexto escolar. Kinesis, v. 15, n. 1, p. 55-66, 1997. 
STRIOTO, L.D.R.; VIEIRA, L.F.; VIEIRA, J.L.L.

WEISS, M. R.; HORN ,T. S.; EBBECK, V. Children's self-perceptions and sources of physical competenceinformation: A cluster analysis. Journal of Sport \& ExerciseP sychology, v. 19, p. 5270, 1997.

YUN, J.; ULRICH, D. A. Perceived and actual physical competence in children with mild mental retardation. A dapted P hysical A ctivity Q uarterly, v. 14, p. 285-297, 1997. 